\title{
OPEN Innovative granular formulation of Metarhizium robertsii microsclerotia and blastospores for cattle tick control
}

\author{
Allan Felipe Marciano ${ }^{1 凶}$, Gabriel Moura Mascarin ${ }^{\Perp}$, Renato Felipe Ferreira Franco ${ }^{3}$, \\ Patrícia Silva Golo ${ }^{1}$, Stefan T. Jaronski ${ }^{4}$, Éverton Kort Kamp Fernandes ${ }^{3}$ \& \\ Vânia Rita Elias Pinheiro Bittencourt ${ }^{1}$ 凶
}

The tick Rhipicephalus microplus poses a serious threat to the cattle industry, resulting in economic losses aggravated by tick resistance to chemical acaricides. Strains of Metarhizium spp., a well-known group of entomopathogenic fungi, can contribute to managing this ectoparasite. We explored two novel granular, microsclerotia- or blastospores-based formulations of Metarhizium robertsii for R. microplus control under semi-field conditions. Fungal persistence in soil was also observed for 336 days. The experiment used pots of Urochloa decumbens cv. Basilisk grass, treated with 0.25 or $0.5 \mathrm{mg}$ of granular formulation $/ \mathrm{cm}^{2}$ ( 25 or $50 \mathrm{~kg} / \mathrm{ha}$ ) applied to the soil surface prior to transferring engorged tick females onto the treated soil. The fungal granules yielded more conidia with subsequent sporulation under controlled indoor conditions than in the outdoor environment, where the levels of fungus rapidly declined over time. Metarhizium-root colonization ranged from 25 to $66.7 \%$ depending on the propagule and rate. Fungal formulations significantly reduced the number of tick larvae during the humid season, reaching at least $64.8 \%$ relative efficacy. Microsclerotia or blastospores-granular formulations of $M$. robertsii can reduce the impact of $R$. microplus, and thus prove to be a promising tool in the control of ticks.

The effective use of microorganisms in biological control programs of arthropod pests depends particularly on the technology for mass production aligned with an effective formulation strategy ${ }^{1}$. Adverse environmental conditions, microbial competitors and host ecology are, however, possible limitations for the successful application of microbial bioproducts targeting either below- or above-ground environments ${ }^{2}$. Among entomopathogenic fungi, the genus Metarhizium is prominent in biological control of a broad range of agricultural pests and constitutes one of the most common active ingredients in mycoinsecticides and mycoacaricides marketed throughout the world ${ }^{3-7}$.

Metarhizium spp. are able to produce different types of propagules, such as conidia and blastospores (BLS) ${ }^{8}$, and some species may produce, by liquid fermentation, compact aggregates of melanized mass of hyphal threads known as microsclerotia (MS), which are structures more resistant to desiccation than fungal conidia ${ }^{9}$, which are important attributes for developing consistently effective bioproducts to control soil-dwelling arthropod pests. BLS are also produced by liquid fermentation, which is convenient for the industry in terms of reduced time, labor and cost ${ }^{4}$. Both MS and BLS are amenable to dry formulations and upon rehydration they can form infective conidia in situ when applied to the environment; in turn, the resultant conidia are able to disperse and infect arthropod hosts ${ }^{4}$. Of particular interest, Metarhizium robertsii, M. brunneum and M. anisopliae not only infect and kill a target pest, but can also colonize the rhizosphere of surrounding plants to their benefit ${ }^{10}$. Bioformulations produced by encapsulation or granulation methods of liquid-culture-grown fungal propagules are gaining attention as an effective environmentally-friendly alternative to chemical insecticides and acaricides,

\footnotetext{
${ }^{1}$ Department of Animal Parasitology, Veterinary Institute, Federal Rural University of Rio de Janeiro, Seropédica, Rio de Janeiro 23897-000, Brazil. 'Laboratory of Environmental Microbiology, Brazilian Agricultural Research Corporation, Embrapa Environment, Jaguariúna, São Paulo 13918-110, Brazil. Institute of Tropical Pathology and Public Health, Federal University of Goiás (UFG), Goiânia, Goiás 74690-900, Brazil. ${ }^{4 J}$ aronski Mycological Consulting, Blacksburg, VA 24060, USA. ${ }^{凶}$ email: allanfmarc@gmail.com; gabriel.mascarin@embrapa.br; vaniabit@gmail.com
} 
as they can enhance fungal efficacy, improve field persistence, and extend product shelf-life. These propagules harbor great promise to replacing aerial conidia, which are still the standard used in fungal biopesticides ${ }^{3}$.

The soil is the site where R. microplus engorged females lay their eggs after dropping from the host, during its non-parasitic life phase, and such behavior is strongly influenced by environmental factors ${ }^{11}$. During this non-parasitic life stage, females, eggs and newly hatched larvae remain on the ground comprising $95 \%$ of the tick population present in the livestock system, which make them easily exposed targets to contact acaricides ${ }^{12}$. Therefore, the application of acaricides targeting eggs and larvae on the pastures would more effectively reach the tick population than applications on infested cattle and also prevent or minimize initial infestation levels on the host cattle. Despite the fact that fungal entomopathogens for tick control have been studied for more than two decades ${ }^{13}$, chemicals remain the chief method to control ticks particularly in Brazil.

Granular formulations for microbial agents are gaining special attention as a cost-effective technology for use with biocontrol agents or bioinoculants in agriculture ${ }^{14}$. When targeting soil-dwelling arthropod pests, small granules could be a viable option to deliver fungal propagules to sites occupied by the target pests. Dry MS-containing granules of M. brunneum, for instance, exhibited superior efficacy in controlling sugarbeet root maggots compared to aerial conidia produced on cereal grains ${ }^{15}$. With proper moisture conditions, the fungus in MS can germinate, grow out, and subsequently sporulate, releasing a large amount of infective conidia in situ where the pest is located. Unlike MS, BLS are environmentally sensitive yeast-like cells that have been tested in granular formulations to control ticks ${ }^{16}$, but a comparison between MS and BLS-based formulations for cattle tick control along with their persistence in soil has not been addressed until this study. Because BLS are more sensitive to drying, storage and adverse environmental conditions, the use of BLS are underexplored, especially regarding soil applications ${ }^{17}$. With technological advances in the production of BLS, these disadvantages are being overcom $\mathrm{e}^{17}$ along with the selection of more resilient strains that are able to withstand environmental constraints ${ }^{18}$.

We investigated the efficacy of granular formulations containing either MS or BLS of M. robertsii strain IP 146 against the tick $R$. microplus in semi-natural pasture conditions. The granular formulation tested in this study was produced based on low-cost matrices of carriers containing MS or BLS for soil applications. We determined the fungal persistence in soil exposed to natural ambient conditions, and further assessed the root colonization by this fungus in the grass plant Urochloa decumbens, extensively employed for grass-fed cattle in Brazil.

\section{Materials and methods}

Experimental design. The study was carried out for 337 consecutive days (Supplementary Fig. S1), January to December, 2019. During this interval, two distinct climatic conditions were encountered: a period of hot days $\left(27.24 \pm 2.10^{\circ} \mathrm{C}\right.$, mean \pm standard deviation) and high volume of precipitation $(9.52 \pm 16.82 \mathrm{~mm})$, and a second season with milder temperatures $\left(22.12 \pm 2.86^{\circ} \mathrm{C}\right)$ and less rain $(2.21 \pm 5.08 \mathrm{~mm})$. The semi-field trial was conducted at Federal Rural University of Rio de Janeiro-UFRRJ, in Rio de Janeiro state, Brazil ( $22^{\circ} 45^{\prime} 54.9^{\prime \prime}$ South and $43^{\circ} 41^{\prime} 57.2^{\prime \prime}$ West, $24 \mathrm{~m}$ a.s.l.). Climatic conditions were monitored using data from a meteorological station. Urochloa decumbens cv. Basilisk seeds (Wolf seeds, Ribeirão Preto, SP, Brazil) were sown in polypropylene pots $(22 \times 24 \times 24 \mathrm{~cm})$. The soil, a Planosol, was autoclaved two cycles at $120^{\circ} \mathrm{C}, 20 \mathrm{~min}$, to eliminate any wild Metarhizium spp., before sowing with seeds and conducting the actual trials, three months later. The temperature of the soil was recorded with an analog thermometer $5 \mathrm{~cm}$ deep in the soil from one pot for each group, daily after treatment. The pots were placed in groups following a completely randomized block design under direct incidence of sun and rain (Supplementary Fig. S2).

Based on previous tests (unpublished data), two concentrations of each granular formulation of microsclerotia (MS) or blastospores (BLS), were established: $0.5 \mathrm{mg} / \mathrm{cm}^{2}$ soil surface (50 kg/ha), subsequently termed MS-G 50 or BLS-G 50, and $0.25 \mathrm{mg} / \mathrm{cm}^{2}(25 \mathrm{~kg} / \mathrm{ha})$, termed hereafter MS-G 25 or BLS-G 25 . There were five treatments with eight grass pots each. Two groups were treated with the granular formulation containing microsclerotia (MS) at each of the described rates. Two other groups were treated with each of the two rates of the granular formulation containing blastospores (BLS). The control group (CTRL) was treated with $0.5 \mathrm{mg} / \mathrm{cm}^{2}(50 \mathrm{~kg} / \mathrm{ha}$ ) of granules formulated without the fungus. The area of the soil surface in pots was $576 \mathrm{~cm}^{2}$, requiring $288 \mathrm{mg}$ or $144 \mathrm{mg}$ of granules per pot for the two rates, respectively.

Granular formulations. The granular formulations, containing either MS or BLS of M. robertsii IP $146^{19}$, were produced at Federal University of Goiás - UFG.

BLS were produced in 250 - $\mathrm{mL}$ flasks containing modified Adámek medium ${ }^{20}$ inoculated with a conidial suspension of $2 \mathrm{~mL}$ at $1.0 \times 10^{8}$ conidia/mL, and agitated for 4 days ${ }^{18}$. Biomass was centrifuged and resuspended in $0.01 \%$ Tween 80 (Labsynth, Brazil) twice; BLS were quantified in a Neubauer chamber and concentration adjusted to $1.0 \times 10^{8} \mathrm{BLS} / \mathrm{mL}$. MS were grown in basal medium ${ }^{21}$ where $10 \mathrm{~mL}$ of the fungal suspension $\left(5.0 \times 10^{7}\right.$ conidia/mL) were added to $90 \mathrm{~mL}$ medium, in $250-\mathrm{mL}$ flasks. The cultures were agitated 4 days ${ }^{15,22}$ after which the biomass was centrifuged and resuspended in $0.01 \%$ Tween 80 twice. The MS concentration was determined according to Jaronski and Jackson ${ }^{15}$ (Supplementary Fig. S3).

Liquid biomass $\left(1.0 \times 10^{4} \mathrm{MS} / \mathrm{mL}\right.$ or $\left.2.0 \times 10^{8} \mathrm{BLS} / \mathrm{mL}\right)$ was mixed with $950 \mathrm{mg}$ of microcrystalline cellulose 101 (Mingtai Chemical, Taiwan) and $50 \mathrm{mg}$ of Psyllium (Natural do Norte, Brazil) for each $\mathrm{mL}$ of biomass, then the material was filtered through a $70-\mathrm{mm}$ diameter filter paper $(14 \mu \mathrm{m}$ porosity; J.PROLAB, São José dos Pinhais, SP, Brazil) under vacuum, homogenized and passed through a 1-mm sieve. Psyllium husk comes from the crushed seeds of the Plantago ovata plant, a herb native to parts of Asia, the Mediterranean, and North Africa ${ }^{23}$. The resultant granules were dried in fluidized bed dryer $\left(1000 \times 1850 \times 630 \mathrm{~mm}, 1.5 \mathrm{~mm}^{3} / \mathrm{min}\right)(\operatorname{model~FBD~} 1.0$, LabMaq, Ribeirão Preto, SP, Brazil) until final moisture reached $5 \pm 1 \%$ (Supplementary Fig. S3). We obtained 
$80 \mathrm{~g}$ of granules with $1.25 \times 10^{4} \mathrm{MS} / \mathrm{g}$ or $2.5 \times 10^{6} \mathrm{BLS} / \mathrm{g}$, which granules were further stored at $6 \pm 1{ }^{\circ} \mathrm{C}$ in sealed 50-mL centrifuged tubes until use (eight days).

Formulation batches of fungal granules were evaluated for viability based on hyphal (myceliogenic) germination and sporogenic germination (conidial production) $)^{8}$ (Supplementary Fig. S4). Each granular formulation $(7 \mathrm{mg})$ was sprinkled onto the surface of Petri plates $(90 \times 15 \mathrm{~mm})$ containing water agar medium $(2 \% \mathrm{w} / \mathrm{v})$ or onto Petri plates $(50 \times 10 \mathrm{~mm})$ containing $10 \mathrm{~g}$ of the same soil (sterile or non-sterile) from the pots. Petri plates were incubated at $25 \pm 1^{\circ} \mathrm{C}$ for seven days and then examined for hyphal germination and conidial production. The viability of conidia was determined by plating suspensions on CTC medium and assessing the number of colony-forming units (CFUs) ${ }^{24}$. Each condition was performed in triplicate and the test was independently repeated at least twice.

Efficacy for Rhipicephalus microplus in semi-field test. The soil in the grass pots was treated by spreading the granules manually as evenly as possible over the surface of previously irrigated soil, and the fungal sporulation was monitored daily. Prior to using in the semi-field trials, we disinfected the cuticles of engorged female ticks ${ }^{25}$; the females were then weighed individually and separated into five classes according to their weights. Forty groups of five females (one tick from each class) were placed on the soil of each grass pot. The first evaluation started with the introduction of engorged females into the pots 8 days after the first fungal application onto the soil (=day 0$)$. In a second evaluation,176 days after the first treatment, engorged females were exposed to the soil to evaluate a possible residual effect from the first treatment. Subsequently, 218 days after the first treatment, a new fungal application was performed (second fungal application) and a third population of engorged females was exposed to the treated soil (third evaluation). After incubation of engorged females on the treated soil, daily inspections were carried out to monitor oviposition and cumulative mortality. As larvae hatched and climbed to the top of the grass blades (Supplementary Fig. S2), they were recovered and quantified to calculate a relative efficacy $(\mathrm{RE})^{25}$. Fungi from dead engorged females were isolated as described in the literature $25,48,49$.

Isolation of fungi. To assess the persistence of $M$. robertsii in the soil, CFUs levels were monitored in soil samples collected one day before the first treatment (day -1) and on days $8,15,30,176,210,225,232,246$, and 336 after the first treatment. Three soil samples from each pot were collected and processed for fungal isolation per the method of Fernandes, et al. ${ }^{26}$.

On days-1, 30,176, and 336, three $U$. decumbens plants were removed carefully from the soil of each grass pot. Three pieces of each plant's roots (approx. $0.6 \mathrm{~mm}$ in length ${ }^{27}$ were plated on CTC medium after surface sterilization, and incubated at $25 \pm 1{ }^{\circ} \mathrm{C}$ for 21 days. The success rate in colonizing $U$. decumbens roots was calculated by counting the number of plants with positive root colonization by $M$. robertsii outgrowth, confirmed based on microscopic examination ${ }^{28}$.

Statistical analysis. All analyses were performed with R Core Team, version 4.0.2 ${ }^{29}$. Tick larval counts were fitted to a generalized linear mixed model (GLMM) with negative binomial distribution (log link function), with the package "glmmTMB" 30 , including tick female exposure (infestation), treatment (fungal formulations and control) and their interaction terms as fixed effects, and block as random effect. Relative efficacy data were fitted to linear mixed model with Gaussian distribution, including evaluation period, formulation and their interaction as fixed factors and block as random effect. Tick survival was analyzed by Kaplan-Meier Survivorship, and survival curves were compared with log-rank test $(P<0.05)$ (package "survival") ${ }^{31}$. Successful colonization of roots by $M$. robertsii was fitted to GLMM with binomial distribution (logit link), including treatment as fixed effect.

Fungal viability of dry granules was fitted to a GLMM with binomial distribution and logit link function. Conidial production was $\log _{10}$-transformed and fitted to a linear mixed model with Gaussian distribution. In both models, fixed effects were attributed to propagule type, substrate type, and their interaction, and observational level and/or experimental date as random effects.

Normally distributed observations with heterogeneous variance in the number of CFUs/g soil were fitted to GLM using the function generalized least square (gls) in "nlme" package ${ }^{32}$, implemented separately for the first fungal application during summer season and the second fungal application during winter season. The model included treatments, evaluation date, and their interaction term as fixed effects, while heterogeneous variance was attributed to each treatment $\times$ time. Correlation between climatic factors recorded during the semi-field experiment and fungal persistence in the soil was performed with non-parametric Spearman rank method. All pairwise multiple comparisons of treatment means were performed with Tukey HSD test $(P<0.05)$, implemented with "emmeans" package ${ }^{33}$.

Ethics statement. Engorged female ticks were obtained from a colony maintained on artificially infested calves in accordance with the regulations of the UFRRJ's Ethics Committee for Animal Experimentation, approved by the protocol \# 9714220419 (Supplementary information). The access to Brazilian genetic heritage was approved by SisGen, protocol \# A420934.

\section{Results}

Conidial yield and fungal viability in granular formulations. The viability of the granules and their conidial production were evaluated under different conditions of substrate and sterility, as well as the viability of the conidia obtained. Granules containing MS or BLS clearly showed the first visible hyphal projections or myceliogenic germination 2 days after incubation on water agar medium. In contrast, on sterile and non-sterile 


\begin{tabular}{|l|l|c|l|l|}
\hline Propagule type & Substrate type & Conidia/g of granules & & \\
\hline \multirow{3}{*}{ MS } & Sterile soil & $153 \times 10^{8} \pm 6.41 \times 10^{8}$ & b & \\
\cline { 2 - 5 } & Non-sterile soil & $11.9 \times 10^{8} \pm 0.50 \times 10^{8}$ & c & * \\
\cline { 2 - 5 } & Water agar & $671 \times 10^{8} \pm 282 \times 10^{8}$ & a & \\
\hline \multirow{3}{*}{ BLS } & Sterile soil & $143 \times 10^{8} \pm 6.01 \times 10^{8}$ & b & \\
\cline { 2 - 5 } & Non-sterile soil & $5.83 \times 10^{8} \pm 0.24 \times 10^{8}$ & c & * \\
\cline { 2 - 5 } & Water agar & $612 \times 10^{8} \pm 25.7 \times 10^{8}$ & a & \\
\hline
\end{tabular}

Table 1. Conidial yield (sporogenic germination) by granular formulations of microsclerotia (MS) or blastospores (BLS) of Metarhizium robertsii (IP 146 strain) on different types of substrates. Means $( \pm$ SE) in the same column followed by different lowercase letters are significantly different among substrate types, and asterisk ${ }^{*}$ ) indicates significant difference between MS and BLS within each substrate type. Pairwise multiple comparisons were conducted with the Tukey HSD test at $P<0.05$.

soil, hyphal growth occurred only 3 and 5 days after incubation, respectively. The granules on water agar and on sterile soil underwent conidiogenesis earlier, i.e., 5 days after incubation in comparison to the granules incubated on non-sterile soil, where sporulation did not take place until the 7 th day.

Conidial production by granular formulations was strongly affected by the type of substrate and type of fungal propagule (interaction: $F_{2,29}=55.04, P<0.0001$ ). For both types of fungal propagule, sporogenic germination of $M$. robertsii IP 146 was always higher on granules inoculated on water agar medium followed by the sterile soil substrate, whereas lower conidial yields were attained by the granules incubated on non-sterile (natural) soil, regardless the propagule type (Table 1).

MS granules produced considerably greater numbers of conidia than BLS granules when inoculated on non-sterile soil, and this superiority was more pronounced when incubated on water agar medium (Table 1). Viability of conidia produced by MS and BLS granules reached $\geq 85 \%$ and was strongly affected by the substrate type $\left(\chi_{2}^{2}=77.82, P<0.0001\right)$, irrespective of propagule type; low viability rates occurred when granules were placed on non-sterile soil (Supplementary Fig. S5). MS granules produced $1.07 \pm 0.07 \times 10^{7}$ and $8.39 \pm 0.67 \times 10^{5}$ conidia/g sterile and non-sterile soil, respectively. The BLS granules reached $1.01 \pm 0.06 \times 10^{7}$ in sterile soil and $4.13 \pm 0.66 \times 10^{5}$ in non-sterile soil.

Efficacy of granular bio-formulations against $\boldsymbol{R}$. microplus in semi-field trials. During the tests, two fungal applications and three evaluations were performed on the tick population, of which two evaluations took place after the first fungal application and a third evaluation was done after the second soil application of the fungal granules. Both concentrations of granular formulations containing MS or BLS significantly decreased the number of tick offspring (reduced numbers of larvae attached to the grass leaves) during the first evaluation period after the first fungal application $\left(\chi_{8}^{2}=78.83, P<0.0001\right)$, relative to the control group (Fig. 1A). After the first fungal application, significant reductions in the number of larvae were achieved with $50 \mathrm{~kg} / \mathrm{ha}$ of BLS-based granules and $25 \mathrm{~kg} / \mathrm{ha}$ of MS-based granules (MS-G 0.25). Conversely, the treatment with $25 \mathrm{~kg} / \mathrm{ha}$ of BLS-based granules (BLS-G 0.25) was not as effective as the other treatments, although it still presented significantly fewer larvae than the control group (Fig. 1A). After five months from the first treatment, a second population of female ticks (second evaluation) was placed into the pots and all groups had a similar number of larvae and no residual effect of $M$. robertsii IP 146 was observed $(P>0.05$, Fig. 1A). A second soil treatment took place followed by a third evaluation of tick population in a different season of the year under a colder and drier climate, but this new treatment with granular formulations was insufficient to reduce larvae outbreak in the grass pots when compared to the control group $(P>0.05$, Fig. 1A).

Nevertheless, when clustering the data across both fungal applications along with the three evaluation periods, $M$. robertsii formulations negatively affected larval load in the pasture $\left(\chi_{4}^{2}=42.77, P<0.0001\right)$ (Fig. 1B). The mean number of tick larvae was lower in the pots treated with the high rate of MS-G $(P<0.0001)$ and BLS-G $(P=0.0001)$, as well as with the lower rate of MS-G $(P=0.0012)$, in comparison to the control group. The treatment with BLS-G $(P=0.3305)$ did not differ from the untreated control, but it also had similar effect from that caused by MS-G $25 P=0.2258$ ) (Fig. 1B).

There was a significant interaction effect between application and evaluation period regarding the relative efficacy against tick larvae $\left(\chi_{6}^{2}=13.23, P=0.0395\right)$. The greatest relative efficacy was achieved in the first exposure of females to the fungus-treated soil (first evaluation), with efficacies of $38.4 \%$ and $64.6 \%$ by BLS-G 25 and MS-G 50, respectively (Table 2). Without reapplying the fungal formulations to grass pots, the relative efficacy was substantially reduced on the second evaluation period in all treatments in comparison to the control groups, with no significant differences among treatments $(P>0.05)$, and averages ranged from $4.1 \%$ (BLS-G 25) to $12.4 \%$ (MS-G 25). After the second application of fungal granules, we documented a pronounced increase in the relative efficacy for the third population of ticks exposed to MS-G 50 in contrast to MS-G 0.25 , and the mean relative efficacy across treatments varied between 1.7 and $23.9 \%$ (Table 2).

During the three exposures of ticks to fungus-treated soil, mortality of engorged females was recorded only after the oviposition period (natural death). Thus, there was no significant effect of $M$. robertsii formulations on tick female survival rates $\left(\chi_{4}^{2}=0.88, P=0.95\right)$ (Supplementary Fig. S6). Only filamentous fungi other than Metarhizium spp. were isolated from tick cadavers found during the experimental period. Thus, the major effect was found on survival of larval ticks. 


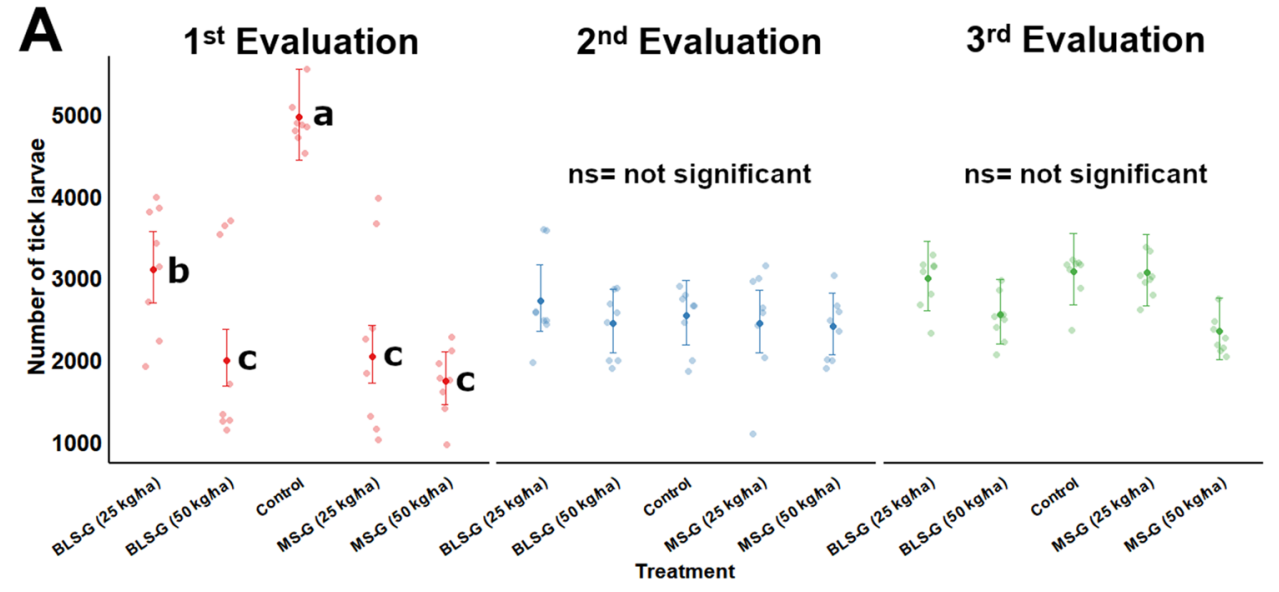

B

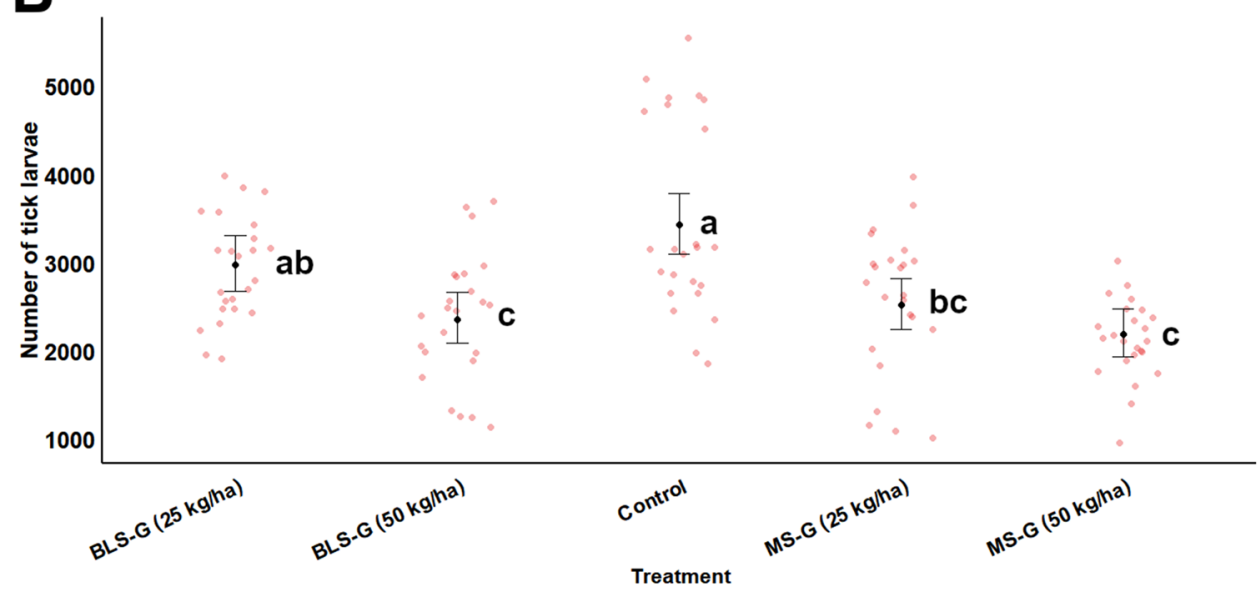

Figure 1. Impact of granular formulations at different rates of Metarhizium robertsii microsclerotia and blastospores on the density of tick larvae on grass pots. Number of Rhipicephalus microplus larvae counted on grass-pots after soil amendments with 50 or $25 \mathrm{~kg} /$ ha of microsclerotia-based granules (MS-G) or blastosporesbased granules (BLS-G). (A) The average number of larvae compared between treatments in three evaluations periods when $R$. microplus female ticks were exposed to the treated environment. In the first and second evaluation, the immediate and residual effect of the first fungal soil application was assessed respectively, in the third evaluation the effect of a new application of the formulations was verified. (B) Overall effect obtained in the number of tick larvae on the three populations of ticks throughout the test period considering the two fungal treatments. Means $(n=8)$ are dots and whiskers represent $95 \%$ confidence intervals. Means followed by distinct letters indicate significant differences (Tukey HSD test $P<0.05$ ). Raw data are shaded dots.

Fungal persistence in the soil and re-isolation from roots. Before and after the treatment of the $U$. decubens pots with the fungal granules, periodic collections of soil and roots of the plant were made for the evaluation of the fungal persistence in the environment. In all treatments, Metarhizium spp. were not recovered from soil or root samples before the application of fungal formulations. After applying the fungal granules to the pots, it was possible to retrieve M. robertsii IP 146 from the soil of all fungal treatments over the experimental span (Fig. 2). There was no recovery of Metarhizium spp. from the soil samples of the untreated controls.

The highest number of CFUs obtained in both seasons was in the first soil sampling soon after the first and the second fungal applications (day 8 and day 225) (Fig. 2). After the first fungal application, during the summer season, the greatest number of CFUs produced by the fungal granules coincided with the highest temperatures and global radiation index registered (Supplementary Fig. S7A), resulting in a positive correlation. On the other hand, the number of CFUs abruptly declined as time progressed (Fig. 2); a high cumulative rainfall occurred, accompanied by high RH indices. CFUs numbers had a negative correlation with these two abiotic variables (Supplementary Fig. S7A). Similarly, the levels of M. robertsii IP 146 invariably decreased after the second fungal application of all fungal formulations tested, but at a slower trend compared with the first fungal application (Fig. 2). The second fungal application was performed on colder days during the winter, which also coincided with the highest number of CFUs of $M$. robertsii recorded during the experimental timeline (Fig. 2). There was a substantial negative correlation between CFUs numbers and the ambient minimum temperature (Supplementary Fig. S7B). Despite the cooler weather in the second experimental period, soil temperatures measured in the grass pots were higher at the beginning of the evaluation than at the end, which resulted in a positive correlation with the high number of CFUs (Supplementary Fig. S7B). After the first fungal application (from day 8 to day 210), 


\begin{tabular}{|l|l|l|l|l|l|}
\hline Evaluation period & Treatment group (kg/ha) & Relative efficacy $\mathbf{~ ( \% ) ~}^{*}$ & $\mathbf{9 5 \%} \mathbf{C I}^{* *}$ & & \\
\hline \multirow{5}{*}{ 1st Evaluation } & MS-G 50 & 64.8 & $54.84,74.7$ & A & a \\
\cline { 2 - 6 } & MS-G 25 & 56.1 & $46.14,66.0$ & A & a \\
\cline { 2 - 7 } & BLS-G 50 & 55.8 & $45.86,65.7$ & A & a \\
\cline { 2 - 7 } & BLS-G 25 & 36.3 & $26.34,46.2$ & B & a \\
\hline \multirow{5}{*}{ 2nd Evaluation } & MS-G 50 & 10.8 & $0.88,20.7$ & A & b \\
\cline { 2 - 7 } & MS-G 25 & 12.4 & $2.51,22.3$ & A & b \\
\cline { 2 - 7 } & BLS-G 50 & 8.7 & $-1.18,18.6$ & A & b \\
\cline { 2 - 7 } & BLS-G 25 & 4.1 & $-5.77,14.0$ & A & b \\
\hline \multirow{5}{*}{ rd Evaluation } & MS-G 0.5 & 23.9 & $13.97,33.8$ & A & b \\
\cline { 2 - 7 } & MS-G 0.25 & 1.7 & $-8.18,11.6$ & B & b \\
\cline { 2 - 7 } & BLS-G 0.5 & 18.8 & $-2.92,28.8$ & AB & b \\
\cline { 2 - 7 } & BLS-G 0.25 & 7.1 & & AB & b \\
\hline
\end{tabular}

Table 2. Relative efficacy (\%) of tick control obtained in three evaluations periods after exposure of Rhipicephalus microplus population to soil treatment with 25 or $50 \mathrm{~kg} / \mathrm{ha}$ of granular formulations containing Metarhizium robertsii microsclerotia (MS-G) or blastospores (BLS-G). In the first and second evaluations, the immediate and residual effect of the first fungal soil application was assessed, respectively, while in the third evaluation the effect of a new application of these fungal treatments was recorded. $\left.{ }^{*}\right)$ Means $(n=8)$ in the same column followed by different uppercase letters are significantly different between treatment groups within the same evaluation period. Means $(n=8)$ in the same column followed by different lowercase letters are significantly different comparing the same treatment group among evaluations periods. Pairwise multiple comparisons were conducted with Tukey HSD test at $P<0.05$. ${ }^{* *}$ Confidence level.

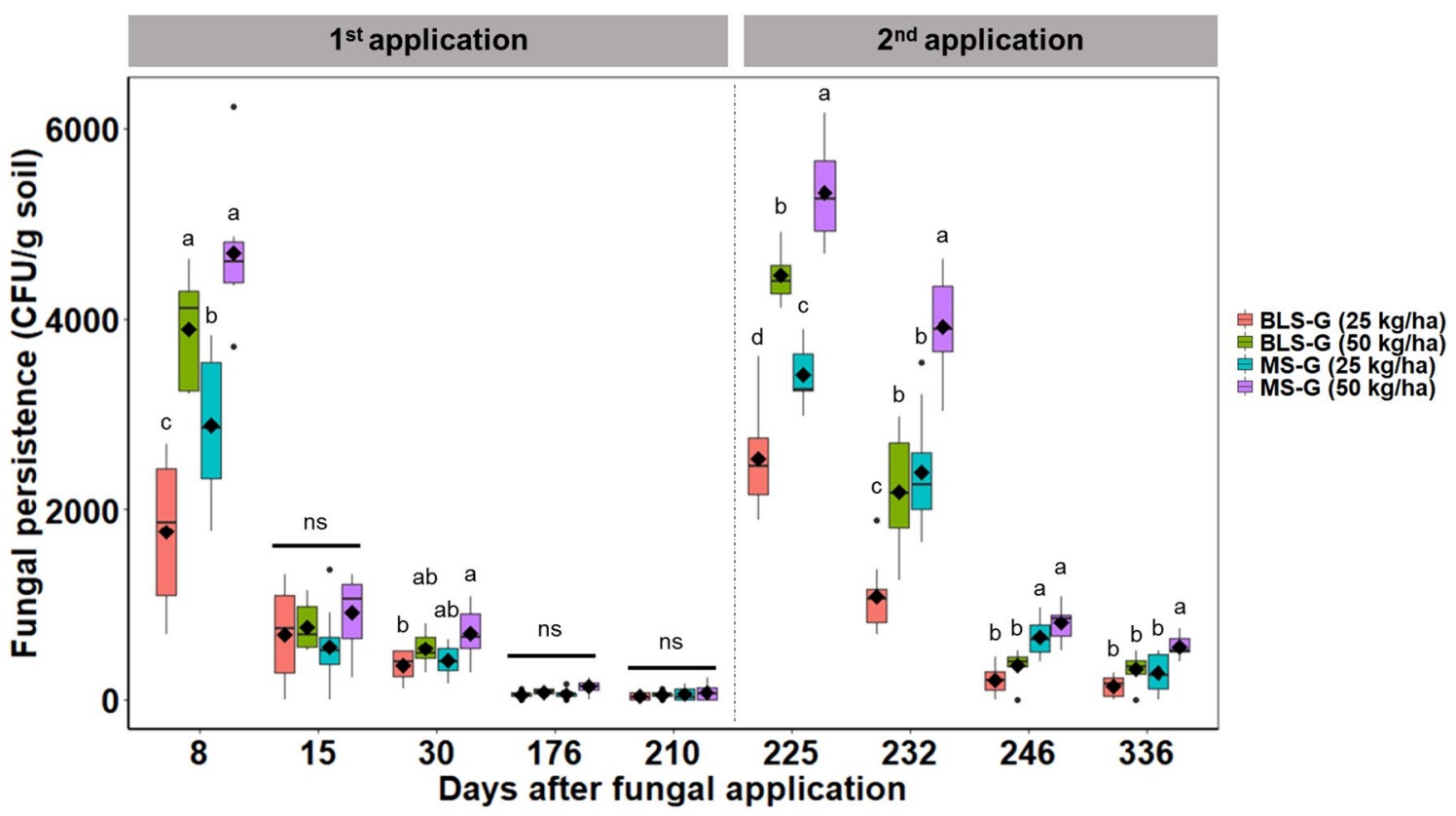

Figure 2. Fungal persistence in the soil of pots treated with Metarhizium robertsii granular formulations. Box plot of $M$. robertsii colony forming units (CFUs) per gram of soil obtained after the 1st and 2nd fungal application under field conditions. Boxes show the median, 25th and 75th percentiles, while error bars show 10th and 90th percentiles. Means $(n=8)$ are black diamonds and followed by distinct letters indicate a significant difference between treatments (Tukey HSD test $P<0.05$ ). BLS-G: granular blastospores formulation; MS-G: granular microsclerotia formulation.

soil levels of $M$. robertsii significantly declined, varying among the fungal formulations. There was a significant interaction effect between treatments and soil sampling dates on fungal persistence (CFUs numbers over time) $\left(F_{12,140}=6.66, P<0.0001\right)$. After the first fungal application, on day 8 , the number of CFUs obtained with the high dose of fungal granules per $\mathrm{cm}^{2}$ (MS-G 50 and BLS-G 50) significantly exceeded the MS-G 25 and BLS-G 25 formulations. Moreover, the soils treated with MS granules at the lower rate (MS-G 0.25) had significantly more CFUs than those treated with the corresponding rate of BLS granules (Fig. 2), reflecting the production of conidia by the MS. Levels of fungus CFUs markedly decreased 15 days after the first fungal application and 


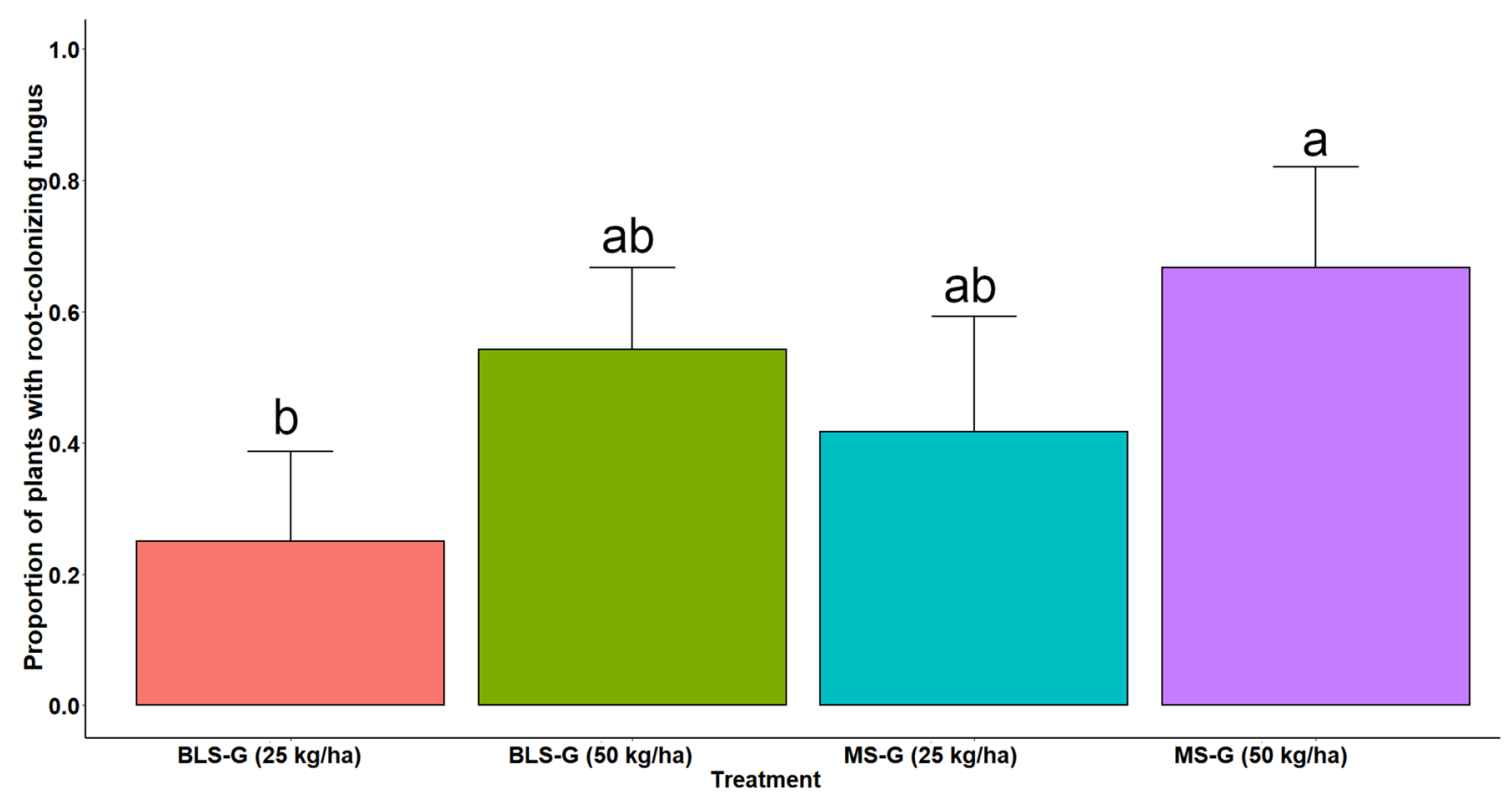

Figure 3. Effect of granular formulations of Metarhizium robertsii on root colonization of potted grass during field experiment of one year. Successful rate in root colonization at the end of the experiment (day 336) after soil amendment with granules containing microsclerotia (MS) or blastospores (BLS) of M. robertsii in granular formulations and applied at two doses (50 and $25 \mathrm{~kg} / \mathrm{ha}$ ). Control group had no root colonization by any naturally-inhabitant Metarhizium species.

equaled the number of CFUs across all treatment groups (Fig. 2). In the following evaluation (day 30), the MS-G 50 treatment presented higher CFUs numbers in the soil than the BLS-G 25 treatment $(P=0.0387)$, even with a declining number of CFUs. The BLS-G 50 and MS-G 25 treatments exhibited similar CFUs numbers and did not differ from the other treatments. In the fourth (day 176) and fifth soil sampling (day 210), the number of CFUs per gram of soil in all treatment groups had significantly decreased in relation to the previous soil samplings, but were statistically similar between these two sampling points (Fig. 2).

Likewise, during the following experimental period, after the second fungal application (from day 225 to day 336), $M$. robertsii levels in pots progressively declined at different rates for the different fungal formulations. There was a significant interaction effect between applications and soil sampling dates regarding the fungal persistence in the soil $\left(F_{9,112}=24.47, P<0.0001\right)$. On day 225 , seven days after the second fungal application, the highest number of $M$. robertsii CFUs was achieved with the highest dose of granules tested (MS-G 50 and BLS-G 50), followed by the treatment with MS-G 0.25 , which had more $M$. robertsii isolated from the soil than the BLS-G 25 group (Fig. 2). On day 232, the MS-G 50 group presented more CFUs of $M$. robertsii in the soil than the groups MS-0.25 ( $P=0.0005)$, BLS-G $50(P=0.0001)$ or BLS-G $25(P=0.0001)$. The BLS-G 50 and MS-G 25 yielded similar CFUs per gram of soil and exhibited significantly more $M$. robertsii CFUs than the group treated with BLS-G 0.25 , with $P=0.0023$ and $P=0.0012$ respectively. In the following soil collection (day 246), CFUs were more numerous in soil treated with MS granules than in those treated with BLS granular formulation; MS-G 25 treatment was significantly different from BLS-G $25(P=0.0006)$ and BLS-G $50(P=0.0250)$, as well as MS-G 50 treatment presented more CFUs per gram of soil than BLS-G $25(P=0.0001)$ and BLS-G $50(P=0.0001)$. In the last assessment (day 336), the MS-G 50 group had significantly higher CFUs per gram of soil than the groups MS-0.25 $(P=0.0165)$, BLS-G $50(P=0.0154)$ and BLS-G $25(P=0.0001)$, which did not differ from each other (Fig. 2$)$.

Regarding $M$. robertsii recovery from $U$. decumbens roots, samples from all groups collected before (day1), and after the first fungal application (day 30) had no fungal growth detected in surface-sterilized roots. Although $M$. robertsii was aseptically isolated from $U$. decumbens roots after the second fungal application, at the end of the experiment (day 336), no Metarhizium sp. was recovered from roots in the control pots. The only difference in the proportion of grass plants with root-colonizing $M$. robertsii among treatments was detected with the higher rate of MS granules (MS-G 50) resulting in $66.7 \pm 9.6 \%$ (mean \pm standard error [SE]) of plants with colonized roots, significantly higher than the treatment with BLS-G 25 , which yielded only $25.0 \pm 8.8 \%$ of plants positive for root colonization $\left(\chi_{3}^{2}=9.46, P=0.0238\right.$ ). The additional fungal treatments (BLS-G 50 and MS-G 25) did not differ in their root colonization success in relation to the other treatments, and their incidence ranged from 41.7 to $54.2 \%$ (Fig. 3).

\section{Discussion}

A large body of literature has supported the use of fungal entomopathogens, like Metarhizium spp. and Beauveria spp., to control ticks and has claimed the importance of developing formulation technologies that improve their effectiveness, persistence in the environment and ease of application ${ }^{4,13,34,35}$. The present study reports the soil amendment of $M$. robertsii granular formulations targeting pastures infested with $R$. microplus ticks under 
semi-field conditions. Long-term persistence of MS and BLS granules applied to the soil was also associated with the ultimate reduction in the number of tick larvae in grass pots.

Even though $M$. robertsii was not re-isolated from tick cadavers due to overgrowth by opportunistic saprobic fungi, the reduction of larvae from fungal treatments is presumed by sub-lethal infection of females which possibly reduced their egg production; in fact, the low egg production from Metarhizium-infected R. microplus females is well documented in the literature ${ }^{18,36-39}$. We also believe that the fungus may have infected eggs and larvae, which are more susceptible life stages than adult ticks ${ }^{40}$. In respect to the application of Metarhizium spp. in pasture environments and their control of $R$. microplus as well as other tick species, the results reported here corroborate previous studies ${ }^{1,25,37,40-42}$ which also showed relative success in controlling this non-parasitic phase of this cattle tick species.

The granular formulation composition, which contains a low-cost matrix of carriers, provides higher viability rates coupled with subsequently higher yields of conidia from both MS and BLS than calcium alginate beads earlier reported for encapsulation of $M$. pemphigi $\mathrm{BLS}^{16}$. In our study, both mycelogenic and sporogenic germination was notably affected by the nature of the substrate, especially when fungal granules were applied on natural (non-sterile) soil cultivated with grass under semi-field conditions versus water agar or sterile soil, with the aim of understanding possible abiotic factors affecting fungal germination and sporulation. MS and BLS granules showed fastest germination rates and greatest conidial production when incubated on water agar medium, which provides the optimal conditions for fungal growth, especially because of the sterile and high humidity microcosm. The high conidial yield achieved with water agar medium may be related to its high, consistent moisture and sterile substrate without competitors, which ensures a steady microclimate when compared to sterile or non-sterile soil substrate in outdoor pots since many field environmental factors can impact the germination of fungal structures ${ }^{43}$. The heterogeneous physical-chemical characteristics of the soil and the presence of microbial competitors, which are part of the resident microbiota in non-sterile soil, may have also lowered the myceliogenic germination, production of conidia and, consequently, the establishment of the strain IP 146 in this environment ${ }^{44-47}$. Even initially, with autoclaved soil, after planting the grass in the pots and exposing them to the outdoor weather conditions, a microbial community re-established itself slowly, but to a lower level than regular non-sterile soil; this was confirmed by the isolation of filamentous fungi other than Metarhizium from the soil samples inoculated on semi-selective CTC medium. The re-established microbial community in the grass-potted soil may have competed with M. robertsii for the same ecological niches. We suggest that the viability and inoculum production of fungal granules are strongly dictated by the environmental conditions and the status of the soil substrate where they were applied, thus impacting on the fungal development and persistence in less controlled and non-homogenous conditions. This situation is confirmed by comparing the concentration of conidia (CFUs) per g of soil obtained in the laboratory and in semi-field conditions in the first soil collection after fungal application. Despite the fact that BLS are considered environmentally sensitive structures less adapted to the soil ${ }^{18}$, and MS comprise resistant-like propagules more suitable for the control of soil-inhabiting arthropods in this environment ${ }^{9,21}$, the BLS granular formulation tested here enabled myceliogenic and sporogenic growth similar to the MS granules. In non-sterile soil, however, MS granules had a significantly higher conidial yield than BLS granules, which suggests a possible advantage of the former fungal propagule to cope with the soil fungistasis in comparison to the latter. This result also corroborates the evidence of natural resilience of Metarhizium spp. MS to adverse abiotic conditions, as previously reported by Jaronski and Jackson ${ }^{15}$. Despite this, it has been observed that, depending on the Metarhizium spp. strain, BLS may be more UV-B tolerant than other propagules ${ }^{48}$. The production of BLS in granular formulations would still be of value, considering their production yield and speed in liquid fermentation but future investigations are necessary to validate the efficiency of their granules.

Our outdoor semi-field experiment revealed a significant decline in fungal persistence in soil over time, an expected behavior for entomopathogenic fungi ${ }^{49}$. This phenomenon is influenced by intrinsic, edaphic, biotic, cultural, and climatic factors ${ }^{49}$. Several climatic variables were recorded during the long-term course of our semifield experiment, and we observed that the two seasons, in which fungal applications took place, diverged in terms of fungal persistence and efficacy against the cattle tick. High temperatures combined with high incidence of rainfall marked the period after the first fungal application, during which the fungi had moderate tick efficacy (36-65\%), and a severe decrease in soil levels of the fungus. During the second application, temperatures were mild, coupled with reduced precipitation, as expected for this locality of Brazil at the onset of winter, and the relative efficacy of tick control was greatly reduced (1.7-23.9\%), but a slower decrease in CFUs/g soil during the initial 7 days after fungus application was recorded (Table 2).

The fungus benefited from the rainy season, with suitable soil moisture for its development, even under high global solar radiation, an important deleterious environmental factor to fungal survival in the environment ${ }^{50}$. Because the pots were covered with grass, the vegetation possibly blocked the direct solar radiation towards the fungal granules deposited on the soil surface, functioning as a buffer from extreme short-term changes in the microclimate and favoring the efficacy of $M$. robertsii ${ }^{51}$. The positive correlation of fungus levels in the soil with the values of temperature, as well as the negative correlation with relative humidity and cumulative rainfall occurred due during the transition from summer to autumn (Supplementary Fig. S7A), in which the days immediately after fungal application and with greater conidial production did not coincide with the climatic factors recorded. After the second fungal application, temperatures and rainfall were significantly reduced. The lack of rain made the soil drier and may have directly precluded propagule germination, sporulation, consequently affected fungus performance. While moisture content in the soil influences the sporulation of granules and interferes with the conidial survivorship ${ }^{52,53}$, moisture also plays an important role in the infection process of the fungus ${ }^{46,54}$.

Similar interpretations regarding the correlation between the density of the fungus and climatic factors must also be considered in the second fungal application. We observed a negative correlation between CFUs density 
and the temperature by the fact that the fungal granules produced more conidia shortly after application, but then fungal persistence substantially decreased in the transition from winter to spring season (warmer and wetter conditions) (Supplementary Fig. S7B).

In the subsurface soil, the longevity of the fungus is related to the ability of this microorganism to establish beneficial relationships with the plant rhizosphere ${ }^{55}$. Here, it was possible to re-isolate M. robertsii IP 146 from grass roots in all fungal treatments after 336 days of the first soil application. The fate of Metarhizium spp. in deeper regions and close to the roots after the inoculum density decreases in the soil surface has been reported by previous studies ${ }^{56,57}$. Accordingly, our study aligned with others to indicate there is a tendency for the fungus to migrate from the most superficial parts to deeper layers and closer to the root system after application to the soil, suggesting that $M$. robertsii IP 146 switches lifestyle from pathogen to endophyte in order to survive and persist in the soil habitat. Fungal granules may allow not only the superficial colonization of the soil but also in deeper layers occupied by the plant rhizosphere, extending the fungal persistence in agricultural ecosystems, as documented before ${ }^{58}$. In fact, $M$. robertsii has already been reported as preferably colonizing pastures and arable lands, possibly due to its wide adaptive skills to such ecological niche ${ }^{59}$. Although we cannot predict how the endophytic root colonization by $M$. robertsii could induce new infections to tick females and their egg masses on the soil surface, this behavior indeed contributes to fungal persistence and probably leads to new foci of infective inoculum derived from sporulated cadavers of other susceptible soil-dwelling arthropod hosts. This hypothesis will remain open but is currently under investigation by our group, as this fungus species is also an important pathogen to several soil-inhabiting insects that are root feeders, such as subterranean termites, wireworms, grubs, and sugarcane spittlebugs in Brazil.

Despite limited fungal persistence in face of environmental challenges, our fungal granular formulations provided a level of tick control. This efficacy was more pronounced after the first fungal application, when the treatments with MS-G at $50 \mathrm{~kg} / \mathrm{ha}, \mathrm{MS}-\mathrm{G}$ at $25 \mathrm{~kg} / \mathrm{ha}$ and BLS-G at $50 \mathrm{~kg} / \mathrm{ha}$ diminished greater than $50 \%$ of tick larvae during the hotter and wetter season. In contrast, a weaker response was observed with BLS-G at $25 \mathrm{~kg} /$ ha, possibly due to the lower resilience of the BLS associated with its lower application rate, which did not yield the sufficient numbers of conidia to reduce tick density. Possibly, an increase of fungal biomass in the granular formulations could improve conidial yields in soils ${ }^{60}$.

Another important observation regarding the residual activity of the fungal formulations on a subsequent tick generation, without reapplication of granules. In our study control of the subsequent generation failed, pointing to the need of a more persistent bioproduct. With the second fungal application, only low efficacy was observed, even with lower temperature and radiation, conditions thought to be favorable to the fungal entomopathogen. It is suggested that, in this case, the scarce rainfall during the winter season may have decreased the ambient humidity (data not shown), thus altering the soil microclimate beneath the grass canopy and affecting the success of the fungal infection on ticks ${ }^{46,54}$.

Our findings highlight for the first time a strategy of using dry granular formulations consisting of either BLS or MS of M. robertsii for soil application to suppress $R$. microplus populations by the tick's population fitness, as well as revealing the soil persistence and grass root-colonization of this fungus in the soil cultivated with grass. Our results advance knowledge on new cost-effective ways to use otherwise environmentally sensitive BLS for soil application and expand the usefulness of MS granules as effective alternative tools to chemical acaricides. This formulation technology can be easily scaled-up to industrial production of fungal propagules (MS and BLS), and has the advantage of inexpensive carrier ingredients. Future challenges to be tackled could focus on the extended fungal persistence after application under a range of conditions beyond the Brazilian situation, and to optimize granule rates applied to the soil with the aim to achieve higher efficacy rates in tick control.

\section{Data availability}

The datasets generated during and/or analyzed during the current study are available from the corresponding author on reasonable request.

Received: 26 October 2020; Accepted: 8 February 2021

Published online: 02 March 2021

\section{References}

1. Behle, R. W., Jackson, M. A. \& Flor-Weiler, L. B. Efficacy of a Granular Formulation Containing Metarhizium brunneum F52 (Hypocreales: Clavicipitaceae) Microsclerotia Against Nymphs of Ixodes scapularis (Acari: Ixoididae). J. Econ. Entomol. 106, 57-63. https://doi.org/10.1603/EC12226 (2013).

2. Vega, F. E. et al. Fungal entomopathogens: new insights on their ecology. Fungal Ecology 2, 149-159. https://doi.org/10.1016/j. funeco.2009.05.001 (2009)

3. Faria, M. R. D. \& Wraight, S. P. Mycoinsecticides and Mycoacaricides: A comprehensive list with worldwide coverage and international classification of formulation types. Biol. Control 43, 237-256. https://doi.org/10.1016/j.biocontrol.2007.08.001 (2007).

4. Mascarin, G. M. et al. Current status and perspectives of fungal entomopathogens used for microbial control of arthropod pests in Brazil. J. Invertebr. Pathol. 165, 46-53. https://doi.org/10.1016/j.jip.2018.01.001 (2019).

5. Thakur, N., Kaur, S., Tomar, P., Thakur, S. \& Yadav, A. N. in New and Future Developments in Microbial Biotechnology and Bioengineering (eds. Rastegari, A. A., Yadav, A. N., \& Yadav, N.) 243-282 (Elsevier, Amsterdam, 2020).

6. Butt, T. M., Coates, C. J., Dubovskiy, I. M. \& Ratcliffe, N. A. in Advances in Genetics Vol. 94 (eds. Lovett, B., \& St. Leger, R. J.) 307-364 (Academic Press, London, 2016).

7. Lacey, L. A. et al. Insect pathogens as biological control agents: Back to the future. J. Invertebr. Pathol. 132, 1-41. https://doi. org/10.1016/j.jip.2015.07.009 (2015).

8. Jackson, M. A., Dunlap, C. A. \& Jaronski, S. T. Ecological considerations in producing and formulating fungal entomopathogens for use in insect biocontrol. Biocontrol 55, 129-145. https://doi.org/10.1007/s10526-009-9240-y (2010). 
9. Jackson, M. A. \& Jaronski, S. T. Production of microsclerotia of the fungal entomopathogen Metarhizium anisopliae and their potential for use as a biocontrol agent for soil-inhabiting insects. Mycol. Res. 113, 842-850. https://doi.org/10.1016/j.mycres.2009.03.004 (2009).

10. Keyser, C. A., Thorup-Kristensen, K. \& Meyling, N. V. Metarhizium seed treatment mediates fungal dispersal via roots and induces infections in insects. Fungal Ecology 11, 122-131. https://doi.org/10.1016/j.funeco.2014.05.005 (2014).

11. Mastropaolo, M., Mangold, A. J., Guglielmone, A. A. \& Nava, S. Non-parasitic life cycle of the cattle tick Rhipicephalus (Boophilus) microplus in Panicum maximum pastures in northern Argentina. Res. Vet. Sci. 115, 138-145. https://doi.org/10.1016/j. rvsc.2017.03.009 (2017).

12. Powell, R. T. \& Reid, T. J. Project tick control [Boophilus microplus, cattle breeds, prevention, resistance, Australia]. Queensland agricultural journal 108, 279-300 (1982).

13. Fernandes, E. K. K., Bittencourt, V. R. E. P. \& Roberts, D. W. Perspectives on the potential of entomopathogenic fungi in biological control of ticks. Exp. Parasitol. 130, 300-305. https://doi.org/10.1016/j.exppara.2011.11.004 (2012).

14. Vemmer, M. \& Patel, A. V. Review of encapsulation methods suitable for microbial biological control agents. Biol. Control 67, 380-389. https://doi.org/10.1016/j.biocontrol.2013.09.003 (2013).

15. Jaronski, S. T. \& Jackson, M. A. Efficacy of Metarhizium anisopliae microsclerotial granules. Biocontrol Sci. Tech. 18, 849-863. https ://doi.org/10.1080/09583150802381144 (2008).

16. Lorenz, S.-C., Humbert, P. \& Patel, A. V. Chitin increases drying survival of encapsulated Metarhizium pemphigi blastospores for Ixodes ricinus control. Ticks and Tick-borne Diseases 11, 101537. https://doi.org/10.1016/j.ttbdis.2020.101537 (2020).

17. Mascarin, G. M., Jackson, M. A., Kobori, N. N., Behle, R. W. \& Delalibera Júnior, İ. Liquid culture fermentation for rapid production of desiccation tolerant blastospores of Beauveria bassiana and Isaria fumosorosea strains. J. Invertebr. Pathol. 127, 11-20. https:// doi.org/10.1016/j.jip.2014.12.001 (2015).

18. Bernardo, C. C. et al. Conidia and blastospores of Metarhizium spp and Beauveria bassiana s.l.: their development during the infection process and virulence against the tick Rhipicephalus microplus. Ticks Tick-Borne Dis. 9, 1334-1342. https://doi.org/10.1016/j. ttbdis.2018.06.001 (2018).

19. Rocha, L. F. N., Inglis, P. W., Humber, R. A., Kipnis, A. \& Luz, C. Occurrence of Metarhizium spp. Central Braz. Soils 53, 251-259. https://doi.org/10.1002/jobm.201100482 (2013).

20. Adamek, L. Submerse cultivation of the fungus Metarrhizium anisopliae (metsch). Folia Microbiol. (Praha) 10, 255-257. https:// doi.org/10.1007/bf02875956 (1965).

21. Mascarin, G. M., Kobori, N. N., de Jesus Vital, R. C., Jackson, M. A. \& Quintela, E. D. Production of microsclerotia by Brazilian strains of Metarhizium spp using submerged liquid culture fermentation. World J. Microbiol. Biotechnol. 30, 1583-1590. https:// doi.org/10.1007/s11274-013-1581-0 (2014).

22. Mascarin, G. M. \& Jaronski, S. T. The production and uses of Beauveria bassiana as a microbial insecticide. World J. Microbiol. Biotechnol. 32, 177. https://doi.org/10.1007/s11274-016-2131-3 (2016).

23. Narladkar, B. W. Projected economic losses due to vector and vector-borne parasitic diseases in livestock of India and its significance in implementing the concept of integrated practices for vector management. Vet. World 11, 151-160. https://doi.org/10.14202/ vetworld.2018.151-160 (2018).

24. Braga, G. U. L., Flint, S. D., Miller, C. D., Anderson, A. J. \& Roberts, D. W. Both solar UVA and UVB radiation impair conidial culturability and delay germination in the entomopathogenic fungus Metarhizium anisopliae. Photochem. Photobiol. 74, 734-739. https://doi.org/10.1562/0031-8655(2001)0740734BSUAUR2.0.CO2 (2001).

25. Marciano, A. F. et al. Metarhizium anisopliae sensu lato (s.l.) oil-in-water emulsions drastically reduced Rhipicephalus microplus larvae outbreak population on artificially infested grass. Med. Vet. Entomol. https://doi.org/10.1111/mve.12445 (2020).

26. Fernandes, É. K. K., Keyser, C. A., Rangel, D. E. N., Foster, R. N. \& Roberts, D. W. CTC medium: a novel dodine-free selective medium for isolating entomopathogenic fungi, especially Metarhizium acridum, from soil. Biol. Control 54, 197-205. https://doi. org/10.1016/j.biocontrol.2010.05.009 (2010).

27. Parsa, S., Ortiz, V. \& Vega, F. E. Establishing fungal entomopathogens as endophytes: towards endophytic biological control. J Vis Exp https://doi.org/10.3791/50360 (2013).

28. Humber, R. A. in Manual of Techniques in Invertebrate Pathology 2nd edn (ed. Lacey, L. A.) 151-187 (Academic Press, London, 2012).

29. R: A Language and Environment for Statistical Computing v. 4.0.2 (R Foundation for Statistical Computing, Vienna, Austria, 2020).

30. Brooks, M. E. et al. glmmTMB balances speed and flexibility among packages for zero-inflated generalized linear mixed modeling. The R Journal 9, 400. https://doi.org/10.3929/ethz-b-000240890 (2017).

31. Terry, M. T. A Package for Survival Analysis in R. R package version 3.2-3 (2020).

32. Pinheiro, J., Bates, D., DebRoy, S., Sarkar, D. \& R Core Team. nlme: Linear and Nonlinear Mixed Effects Models. R package version 3.1-149 (2020)

33. Russell, L. emmeans: Estimated Marginal Means, aka Least-Squares Means. R package version 1.4.8 (2020).

34. Samish, M., Ginsberg, H. \& Glazer, I. Biological control of ticks. Parasitology 129, S389-S403. https://doi.org/10.1017/S003118200 4005219 (2004).

35. Beys-da-Silva, W. O. et al. Updating the application of Metarhizium anisopliae to control cattle tick Rhipicephalus microplus (Acari: Ixodidae). Exp. Parasitol. 208, 107812. https://doi.org/10.1016/j.exppara.2019.107812 (2020).

36. FernándeZ-SalaS, A. et al. Acaricidal activity of Metarhizium anisopliae isolated from paddocks in the Mexican tropics against two populations of the cattle tick Rhipicephalus microplus. Med. Vet. Entomol. 31, 36-43. https://doi.org/10.1111/mve.12203 (2017).

37. Mesquita, E. et al. Efficacy of a native isolate of the entomopathogenic fungus Metarhizium anisopliae against larval tick outbreaks under semifield conditions. Biocontrol https://doi.org/10.1007/s10526-020-10006-1 (2020).

38. Perinotto, W. M. S. et al. Susceptibility of different populations of ticks to entomopathogenic fungi. Exp. Parasitol. 130, 257-260. https://doi.org/10.1016/j.exppara.2011.12.003 (2012).

39. Camargo, M. G. et al. Effect of oil-based formulations of acaripathogenic fungi to control Rhipicephalus microplus ticks under laboratory conditions. Vet. Parasitol. 188, 140-147. https://doi.org/10.1016/j.vetpar.2012.03.012 (2012).

40. Gindin, G., Samish, M., Zangi, G., Mishoutchenko, A. \& Glazer, I. The susceptibility of different species and stages of ticks to entomopathogenic fungi. Exp. Appl. Acarol. 28, 283-288. https://doi.org/10.1023/A:1025379307255 (2002).

41. Ojeda-Chi, M. M., Rodriguez-Vivas, R. I., Galindo-Velasco, E. \& Lezama-Gutiérrrez, R. Laboratory and field evaluation of Metarhizium anisopliae (Deuteromycotina: Hyphomycetes) for the control of Rhipicephalus microplus (Acari: Ixodidae) in the Mexican tropics. Vet. Parasitol. 170, 348-354. https://doi.org/10.1016/j.vetpar.2010.02.022 (2010).

42. Wassermann, M., Selzer, P., Steidle, J. L. M. \& Mackenstedt, U. Biological control of Ixodes ricinus larvae and nymphs with Metarhizium anisopliae blastospores. Ticks Tick-Borne Dis. 7, 768-771. https://doi.org/10.1016/j.ttbdis.2016.03.010 (2016).

43. Jackson, A. M., Whipps, J. M. \& Lynch, J. M. Effects of temperature, $\mathrm{pH}$ and water potential on growth of four fungi with disease biocontrol potential. World J. Microbiol. Biotechnol. 7, 494-501. https://doi.org/10.1007/BF00303376 (1991).

44. Ghini, R. \& Morandi, M. A. B. Biotic and abiotic factors associated with soil suppressiveness to Rhizoctonia solani. J Sci. Agric. 63, 153-160. https://doi.org/10.1590/S0103-90162006000200007 (2006).

45. Singh, B. K., Dawson, L. A., Macdonald, C. A. \& Buckland, S. M. Impact of biotic and abiotic interaction on soil microbial communities and functions: a field study. Appl. Soil. Ecol. 41, 239-248. https://doi.org/10.1016/j.apsoil.2008.10.003 (2009). 
46. Jaronski, S. T. Ecological factors in the inundative use of fungal entomopathogens. Biocontrol 55, 159-185. https://doi.org/10.1007/ s10526-009-9248-3 (2010).

47. Parsa, S., Ortiz, V., Gómez-Jiménez, M. I., Kramer, M. \& Vega, F. E. Root environment is a key determinant of fungal entomopathogen endophytism following seed treatment in the common bean, Phaseolus vulgaris. Biol. Control 116, 74-81. https://doi. org/10.1016/j.biocontrol.2016.09.001 (2018).

48. Corval, A. R. C. et al. UV-B tolerances of conidia, blastospores, and microsclerotia of Metarhizium spp entomopathogenic fungi. J. Basic Microbiol. https://doi.org/10.1002/jobm.202000515 (2020).

49. Scheepmaker, J. W. A. \& Butt, T. M. Natural and released inoculum levels of entomopathogenic fungal biocontrol agents in soil in relation to risk assessment and in accordance with EU regulations. Biocontrol Sci. Tech. 20, 503-552. https://doi.org/10.1080/09583 150903545035 (2010).

50. Fernandes, É. K. K., Rangel, D. E. N., Braga, G. U. L. \& Roberts, D. W. Tolerance of entomopathogenic fungi to ultraviolet radiation: a review on screening of strains and their formulation. Curr. Genet. 61, 427-440. https://doi.org/10.1007/s00294-015-0492-Z (2015).

51. Samish, M. et al. Efficacy of the entomopathogenic fungus Metarhizium brunneum in controlling the tick Rhipicephalus annulatus under field conditions. Vet. Parasitol. 206, 258-266. https://doi.org/10.1016/j.vetpar.2014.10.019 (2014).

52. Ekesi, S., Maniania, N. K. \& Lux, S. A. Effect of soil temperature and moisture on survival and infectivity of Metarhizium anisopliae to four tephritid fruit fly puparia. J. Invertebr. Pathol. 83, 157-167. https://doi.org/10.1016/S0022-2011(03)00069-7 (2003).

53. Zhang, X., Lei, Z., Reitz, S. R., Wu, S. \& Gao, Y. Laboratory and greenhouse evaluation of a granular formulation of Beauveria bassiana for control of western flower thrips, Frankliniella occidentalis. Insects 10, 58. https://doi.org/10.3390/insects10020058 (2019).

54. Muniz, E. R. et al. Efficacy of Metarhizium anisopliae conidia in oil-in-water emulsion against the tick Rhipicephalus microplus under heat and dry conditions. Biocontrol 65, 339-351. https://doi.org/10.1007/s10526-020-10002-5 (2020).

55. Liao, X., O’Brien, T. R., Fang, W. \& St. Leger, R. J. The plant beneficial effects of Metarhizium species correlate with their association with roots. Appl. Microbiol. Biotechnol. 98, 7089-7096. https://doi.org/10.1007/s00253-014-5788-2 (2014).

56. Bruck, D. J. in The Ecology of Fungal Entomopathogens (eds. Roy, H. E. et al.) 103-112 (Springer Netherlands, Berlin 2010).

57. Yang, H. et al. Persistence of Metarhizium (Hypocreales: Clavicipitaceae) and Beauveria bassiana (Hypocreales: Clavicipitaceae) in tobacco soils and potential as biocontrol agents of Spodoptera litura (Lepidoptera: Noctuidae). Environ. Entomol. 48, $147-155$. https://doi.org/10.1093/ee/nvy161 (2018).

58. Barelli, L., Waller, A. S., Behie, S. W. \& Bidochka, M. J. Plant microbiome analysis after Metarhizium amendment reveals increases in abundance of plant growth-promoting organisms and maintenance of disease-suppressive soil. PLoS ONE 15, e0231150. https ://doi.org/10.1371/journal.pone.0231150 (2020).

59. Bidochka, M. J., Kamp, A. M., Lavender, T. M., Dekoning, J. \& De Croos, J. N. A. Habitat Association in Two Genetic Groups of the Insect-Pathogenic Fungus Metarhizium anisopliae: Uncovering Cryptic Species?. Appl. Environ. Microbiol. 67, 1335-1342. https://doi.org/10.1128/AEM.67.3.1335-1342.2001 (2001).

60. Clifton, E. H., Gardescu, S., Behle, R. W. \& Hajek, A. E. Asian longhorned beetle bioassays to evaluate formulation and doseresponse effects of Metarhizium microsclerotia. J. Invertebr. Pathol. 163, 64-66. https://doi.org/10.1016/j.jip.2019.03.005 (2019).

\section{Acknowledgements}

This study was financed in part by the Coordenação de Aperfeiçoamento de Pessoal de Nível Superior of Brasil (CAPES) - Finance Code 001, Conselho Nacional de Desenvolvimento Científico e Tecnológico (CNPq), Fundação Carlos Chagas Filho de Amparo à Pesquisa do Estado do Rio de Janeiro (FAPERJ) and Fundação de Amparo à Pesquisa do Estado de Goiás (FAPEG/PRONEM/CNPq \#201710267000515). CNPq provided the grant PQ 306319/2018-7 for E.K.K.F. and PQ 302985/2019-0 for V.R.E.P.B. We thank Marcos Daniel Filgueiras Gomes, from UFG, for technical assistance in preparing the fungal granules, and the entire team from Laboratório de Controle Microbiano (LCM-UFRRJ) for their support in the field evaluations.

\section{Author contributions}

A.F.M. and E.K.K.F. conceived the experiment; A.F.M. and R.F.F.F. conducted the experiment; A.F.M. and G.M.M. analyzed the results, prepared a manuscript draft and edited the manuscript; G.M.M., P.S.G., S.T.J., E.K.K.F. revised the manuscript for technical and scientific accuracy; E.K.K.F., V.R.E.P.B. acquired funding and supervised the project. All authors approved the manuscript.

\section{Competing interests}

The authors declare no competing interests.

\section{Additional information}

Supplementary Information The online version contains supplementary material available at https://doi. org/10.1038/s41598-021-84142-8.

Correspondence and requests for materials should be addressed to A.F.M., G.M.M. or V.R.E.P.B.

Reprints and permissions information is available at www.nature.com/reprints.

Publisher's note Springer Nature remains neutral with regard to jurisdictional claims in published maps and institutional affiliations.

Open Access This article is licensed under a Creative Commons Attribution 4.0 International License, which permits use, sharing, adaptation, distribution and reproduction in any medium or format, as long as you give appropriate credit to the original author(s) and the source, provide a link to the Creative Commons licence, and indicate if changes were made. The images or other third party material in this article are included in the article's Creative Commons licence, unless indicated otherwise in a credit line to the material. If material is not included in the article's Creative Commons licence and your intended use is not permitted by statutory regulation or exceeds the permitted use, you will need to obtain permission directly from the copyright holder. To view a copy of this licence, visit http://creativecommons.org/licenses/by/4.0/.

(C) The Author(s) 2021 